\title{
SIMULAÇÃO DO PROCESSO DE HIDROTRATAMENTO CATALÍTICO DE FRAÇÕES DE PETRÓLEO EM LEITO FIXO
}

\author{
V. M. C. ALVES ${ }^{1}$, J. J. N. ALVES ${ }^{1}$, A. T. P. NETO ${ }^{1}$, B. V. SOUSA ${ }^{1}$ \\ ${ }^{1}$ Universidade Federal de Campina Grande, Unidade Acadêmica de Engenharia Química \\ E-mail para contato: victormanuelcunhaalves@gmail.com
}

\begin{abstract}
RESUMO - O processo de hidrotratamento (HDT) tem o objetivo de reduzir a concentração de enxofre, oxigênio e nitrogênio contidos nos hidrocarbonetos combustíveis. Este trabalho tem como objetivo desenvolver a modelagem matemática do processo de HDT, incluindo os fenômenos de transferência entre as fases gás-líquido e líquido-sólido e na cinética das reações, resultando um sistema diferencial-algébrico. O modelo matemático foi implementado em um código de cálculo para prever o comportamento de reatores de HDT no Software MATLAB. Os resultados previstos com o modelo foram validados com dados de planta piloto, considerando-se apenas a reação de dessulfurização. Obteve-se os perfis de pressão parcial de $\mathrm{H}_{2}$ e $\mathrm{H}_{2} \mathrm{~S}$ na fase gasosa ao longo do reator, perfil de concentração de enxofre na fase liquida, além da concentração de hidrogênio e sulfeto de hidrogênio no seio da fase liquida e na interface com a superfície catalítica. Os resultados previstos corroboram com os dados experimentais da literatura.
\end{abstract}

\section{INTRODUÇÃO}

Por mais de três décadas, as refinarias em todo o mundo têm vindo a implementar vários projetos em suas instalações para acomodar uma variedade de regulamentos para melhorar a qualidade dos combustíveis de transporte, a fim de reduzir as emissões dos veículos. Uma das principais áreas de interesse tem sido a redução do enxofre no diesel a níveis muito baixos. Desde meados de 2006, o teor máximo de enxofre do diesel nos EUA tem sido limitado a 15 ppm em peso. Regulamentos semelhantes estão em vigor ou estão em diferentes fases de implementação em muitos países (Bonfá, 2011). Com isso, o aumento da demanda mundial por combustíveis limpos requer o melhoramento da tecnologia do processo de hidrotratamento (Schweitzer et al., 2010).

Atualmente no Brasil, o grande desafio se dá exatamente na adequação do parque de refino já existente às exigências ambientais que estão vigentes e cada vez mais rigorosas. A correta aplicação deste processo reduz a taxa de emissão de óxidos extremamente nocivos à atmosfera e ao meio ambiente em geral, como o $\mathrm{SO}_{2}$ e o $\mathrm{SO}_{3}$.

Este trabalho tem por objetivo o desenvolvimento de modelos matemáticos capazes de prever o comportamento de reatores de hidrotratamento (HDT), levando-se em consideração a transferência entre as fases gás-líquido e líquido-solido envolvidas no processo, além da cinética das reações presentes. O modelo matemático foi implementado em um código de cálculo no software MATLAB®. 


\section{MODELAGEM MATEMÁTICA}

O modelo matemático baseia-se nos princípios de conservação da massa e energia. Admitiu-se um reator perfeitamente isolado termicamente, o que significa que não há troca de calor com o ambiente. Assumiu-se ainda escoamento tubular de fluxo pistonado.

\subsection{Balanços de Massa}

Os balanços de massa foram obtidos com as mesmas considerações de Korsten e Hoffmann (1996):

- Há somente uma reação, a dessulfurização;

- Desconsidera-se a vaporização da carga;

- Ausência de gradientes radiais de temperatura e concentração;

- Reações recorrentes apenas na superfície do catalisador e em seus poros;

- Coeficientes estequiométricos ajustados para representar o real consumo de hidrogênio na planta;

- Ausência de desativação catalítica;

- Não há perda de carga ao longo do processo;

- Temperatura a homogênea entre as três fases envolvidas no processo;

- A velocidade da fase líquida é constante ao longo de todo o sistema reacional;

- Velocidade da fase gasosa varia, dependendo da pressão e/ou temperatura;

- Despreza-se o hidrocraqueamento, e a formação de hidrocarbonetos leves.

O balanço para os componentes na fase gasosa, considerando o equilíbrio com a fase líquida dado pela lei de Henry, que no caso são $\mathrm{H}_{2}$ e $\mathrm{H}_{2} \mathrm{~S}$, é dado pela Equação 1:

$$
\frac{u^{G}}{R T} \frac{\delta_{P i}{ }^{G}}{\delta z}+k_{i}{ }^{L} a^{L}\left(\frac{P i^{G}}{H_{i}}-C i^{L}\right)=0
$$

Para os componentes na fase líquida, o balanço é dado pela Equação 2:

$$
\frac{u^{L}}{R T} \frac{\delta_{C i}{ }^{L}}{\delta z}-k_{i}{ }^{L} a^{L}\left(\frac{P i^{G}}{H_{i}}-C i^{L}\right)+k_{i}{ }^{S} a^{S}\left(C i^{L}-C i^{S}\right)=0
$$

Caso o componente da fase líquida seja não-volátil, não existirá o termo de transferência de massa entre as fases liquida e gasosa, de modo que, para estes não voláteis, o balanço reduz-se a Equação 3:

$$
\frac{u^{L}}{R T} \frac{\delta_{C i}{ }^{L}}{\delta z}+k_{i}^{S} a^{S}\left(C i^{L}-C i^{S}\right)=0
$$

Todos os componentes em todas as fases estão presentes na reação de dessulfurização, que ocorre na superfície do catalisador, sendo consumidos ou formados. A Equação 4 contém a taxa de reação de dessulfurização $r_{h d s}$. Na pratica a efetividade catalítica varia entre $0,057 \mathrm{e}$ 1 (Li et al.,1995; Scamangas e Marangozis,1982; Weiss et al., 1987). Neste trabalho assumiuse efetividade catalítica máxima (igual a 1). Como não há partículas inertes, o fator de diluição é igual a 1 .

$$
k_{i}^{s} a^{s}\left(C i^{L}-C i^{S}\right)=-\rho_{l} \cdot \xi \cdot \eta \cdot \sigma_{H D S} \cdot r_{h d s}
$$



literatura.

Os parâmetros do modelo foram calculados a partir de correlações disponíveis na

A densidade é calculada a partir da correlação de Standing - Katz (Ahmed, 1989), com unidades no sistema inglês (libra/pé3), descrita na Equação 5:

$$
\rho(P, T)=\rho_{0}+\Delta \rho_{p}-\Delta \rho_{T}
$$

Para o cálculo de $\Delta \rho_{p}$, influência da pressão na densidade, tem-se a Equação 6:

$$
\Delta \rho_{p}=\left[0,167+16,181 \cdot 10^{-0,0425 \times \rho_{0}}\right] \cdot\left[\frac{P}{1000}\right]-0,01 \cdot\left[0,299+263 \cdot 10^{-0,0603 \rho_{0}}\right] \cdot\left[\frac{P}{1000}\right]^{2}
$$

Para a levar em consideração o efeito da temperatura $\left({ }^{\circ} \mathrm{R}\right)$, tem-se a Equação 7:

$$
\begin{aligned}
& \Delta \rho_{T}=\left[0,0133+152,4 \cdot\left(\rho_{0}+\Delta \rho_{p}\right)^{-2,45}\right] \cdot[T-520] \\
&-\left[8.1 \cdot 10^{-6}-0,0622 \cdot 10^{-0,764\left(\rho_{0}+\Delta \rho_{p}\right)}\right] \cdot[\mathrm{T}-520]^{2}
\end{aligned}
$$

O coeficiente de Henry que aparece nos balanços de massa, é determinado a partir da Equação 8 a seguir:

$$
H i=\frac{V_{n}}{\lambda i \cdot \rho_{L}}
$$

A solubilidade do $\mathrm{H}_{2}$, é dada pela Equação 9:

$$
\lambda_{H}=a 0+a 1 \cdot T+a 2 \cdot \frac{T}{\rho_{20}}+a 3 \cdot T^{2}+a 4 \cdot \frac{1}{\rho^{2}{ }_{20}}
$$

Com a temperatura em ${ }^{\circ} \mathrm{C}$ e as constantes $\mathrm{a}_{\mathrm{i}}$ :

$$
\begin{aligned}
& a_{0}=-0,559729 \\
& a_{1}=-0,42947 \cdot 10^{-3} \\
& a_{2}=3,07539 \cdot 10^{-3} \\
& a_{3}=1,94593 \cdot 10^{-6} \\
& a_{4}=0,835783
\end{aligned}
$$

A solubilidade do sulfeto de hidrogênio é dada pela Equação 10:

$$
\lambda_{H 2 S}=e^{3,3670-0,008470 \cdot T}
$$

Para a determinação dos coeficientes de transferência de massa gás-líquido, utiliza-se a Equação 11 proposta por Goto e Smith (1975):

$$
\frac{k_{i}^{L} a_{L}}{D_{i}^{L}}=\alpha_{1} \cdot\left(\frac{G_{l}}{\mu_{L}}\right)^{\alpha_{2}} \cdot\left(\frac{\mu_{L}}{D_{i}^{L} \rho_{L}}\right)^{\frac{1}{2}}
$$

$\alpha_{2}$ e $\alpha_{1}$ são constantes com valores de 0.4 e $7 \mathrm{~cm}^{-1,6}$, respectivamente.

A determinação do coeficiente de transferência de massa é dependente da viscosidade dinâmica da mistura, que por sua vez depende da temperatura. Esta dependência é dada pela 
Equação 12 publicada por Reid et al (1987), sendo T a temperatura em ${ }^{\circ} \mathrm{R}$ e API o grau API do diesel:

$$
\begin{aligned}
& \mu=3,141 \cdot 10^{10}(T-460)^{-3,444} \cdot\left[\log _{10}(A P I)\right]^{a} \\
& a=10,313 \cdot\left[\log _{10}(T-460)\right]-36,447
\end{aligned}
$$

Assumindo total diluição dos solutos, a difusividade pode ser estimada pela correlação de Tyn-Calus como proposto por Reid et al. (1987):

$$
D_{i}^{L}=8,93 \cdot 10^{-8} \cdot \frac{\vartheta_{L}^{0,267}}{\vartheta_{i}^{0,433}} \cdot \frac{T}{\mu_{L}}
$$

Com T em K. O volume molar do soluto e do solvente, podem ser estimados através da seguinte correlação, descrita por Perry e Green (1984):

$$
\vartheta=0,285 \cdot \vartheta_{c}^{1,048}
$$

Os volumes críticos específicos do $\mathrm{H}_{2}$ e $\mathrm{H}_{2} \mathrm{~S}$ são tabelados, entretanto o do óleo foi determinado através da correlação de Riazi-Daubert proposta por Ahmed (1989):

$$
v_{c}^{m}=7,5214 \cdot 10^{-3} \cdot T_{\text {medio }}^{0,2896} \cdot \rho_{15,6^{\circ} \mathrm{C}}^{-0,7666}
$$

A conversão do volume molar crítico para volume crítico se dá apenas multiplicando o primeiro pela massa molar do composto, resultando no segundo.

Para o coeficiente de transferência de massa líquido-sólido, pode ser utilizada a equação de van Krevelen-Krekels, uma vez que essa transferência seja em regime laminar (Froment e Bischoff, 1990):

$$
\frac{k_{i}^{S}}{D_{i}^{L} a^{S}}=1,8 \cdot\left(\frac{G_{L}}{a^{S} \mu_{L}}\right)^{0,5} \cdot\left(\frac{\mu_{L}}{\rho_{L} \cdot D_{i}^{L}}\right)^{\frac{1}{3}}
$$

A área específica do catalisador á dada pela Equação 18. O diâmetro da partícula foi de $1,72 \mathrm{~mm}$, e a porosidade do leito catalítico igual a 0,5 .

$$
a^{S}=\frac{6}{d p}(1-\epsilon)
$$

\subsection{Modelo Cinético Reacional}

No modelo desenvolvido, as seguintes reações de dessulfurização foram levadas em consideração:

Dissulfurados: $\mathrm{RS}_{2}+3 \mathrm{H}_{2} \rightarrow 2 \mathrm{RH}+2 \mathrm{H}_{2} \mathrm{~S}$

Mercaptanas: $\mathrm{R}-\mathrm{SH}+\mathrm{H}_{2} \rightarrow \mathrm{RH}+\mathrm{H}_{2} \mathrm{~S}$

Sulfurados: $\quad \mathrm{R}_{2} \mathrm{~S}+2 \mathrm{H}_{2} \rightarrow 2 \mathrm{RH}+\mathrm{H}_{2} \mathrm{~S}$

Tiofeno: $\quad \mathrm{C}_{4} \mathrm{H}_{4} \mathrm{~S}+4 \mathrm{H}_{2} \rightarrow \mathrm{C}_{4} \mathrm{H}_{10}+\mathrm{H}_{2} \mathrm{~S}$ 
As quais foram agrupadas em um único lump. Ao agrupar todas as reações de enxofre em um único lump, a reação genérica é:

$$
\sigma_{1} R-S+\sigma_{2} H_{2} \rightarrow \sigma_{3} R-C H+\sigma_{4} H_{2} S
$$

$\mathrm{R}-\mathrm{S}$ representa os compostos sulfurados, e R - CH os dessulfurados. Segundo Korsten e Hoffmann (1996), para os compostos sulfurados, o coeficiente estequiométrico é -1; Para o hidrogênio, corresponde a -15 , e para o sulfeto de hidrogênio, 9.

A cinética da dessulfurização baseia-se no modelo de Langmuir - Hinshelwood, sendo considerada irreversível (Chowdhury, Pedernera et al, 2002)

$$
r_{H D S}=\frac{k_{H D S} C_{\text {sulfurado }} C^{0.5}{ }_{H 2}}{1+k_{\text {ads }}^{H 2 S} C_{H 2 S}}
$$

\section{RESULTADOS}

Simulou-se o processo e validou-se o modelo matemático do mesmo com um código de cálculo desenvolvido no software MATLAB®. Foram traçados perfis de concentração de enxofre na carga e $\mathrm{H}_{2} \mathrm{~S}$ formado ao longo do reator. Obteve-se também os perfis das pressões parciais dos componentes gasosos ao longo do reator. A Figura 1 apresenta o perfil de dessulfurização da carga de entrada, comprovando a eficácia do modelo.

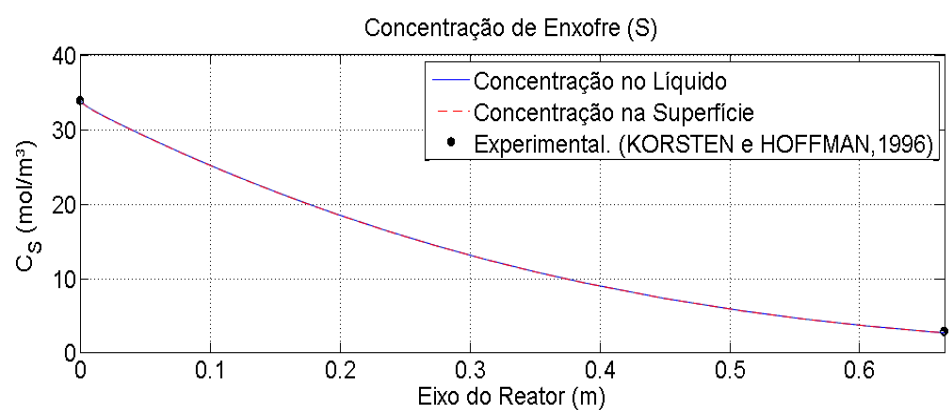

Figura 1 - Concentração de Enxofre.

A partir da Figura 1, observa-se que as curvas que descrevem as concentrações presentes no líquido e na superfície catalítica ficam sobrepostas, o que significa que a taxa de transferência de massa de componentes sulfurados é muito maior do que a sua respectiva taxa de reação.

A Figura 2 apresenta o comportamento da concentração de $\mathrm{H}_{2} \mathrm{~S}$ ao longo do reator. É possível observar que diferentemente do conjunto de curvas apresentadas na Figura 1, há diferença entre a concentração presente no seio do líquido e na interface com a superfície catalítica. Segundo Neto (2013), isso se dá porque a taxa de formação do $\mathrm{H}_{2} \mathrm{~S}$ é maior do que a dessorção que ocorre na superfície. O rápido crescimento da curva é justificável devido à alta atividade catalítica do processo. Para a concentração de hidrogênio, na Figura 3, há um decaimento inicial, uma vez que este está sendo consumido pela reação. 


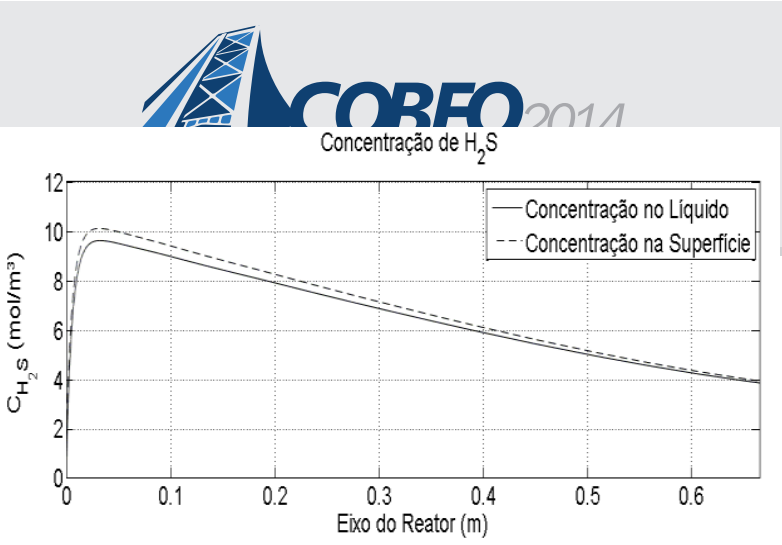

Figura 2- Concentração de sulfeto de hidrogênio.
19 a 22 de nutubro de 2014

Concentração de $\mathrm{H}_{2}$

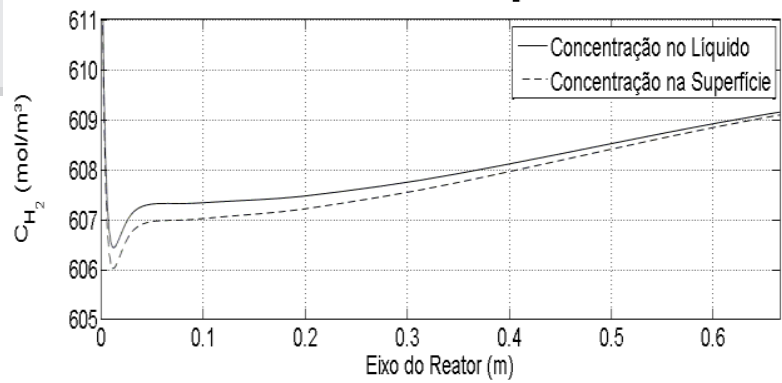

Figura 3 - Concentração do hidrogênio.

Observa-se na Figura 3 de que como o hidrogênio é reagente, ele deveria ter o comportamento continuamente decrescente ao longo do reator. Todavia, ao fazer uma análise mais criteriosa, ocorre que devido à alta quantidade de hidrogênio injetada inicialmente no reator, a relação entre hidrogênio consumido e fornecido garante o fato de que a quantidade disponível no sistema deste é muito maior do que a consumida pela hidrodessulfurização, o que torna após a realização da reação, a retomada do crescimento da concentração de hidrogênio. Além disso, é necessária uma quantidade considerável de hidrogênio em excesso, para que este dilua o $\mathrm{H}_{2} \mathrm{~S}$ gerado, a fim de evitar que este último iniba a reação de HDS. As Figuras 4 e 5 apresentam o perfil das pressões parciais de $\mathrm{H}_{2} \mathrm{~S}$ e $\mathrm{H}_{2}$ em suas formas gasosas, de maneira respectiva. É válida a observação de que ambas as curvas estão condizentes com os perfis previstos na literatura (Korsten e Hoffman, 1996) e com a cinética envolvida (Chowdhury, Pedernera et al, 2002).

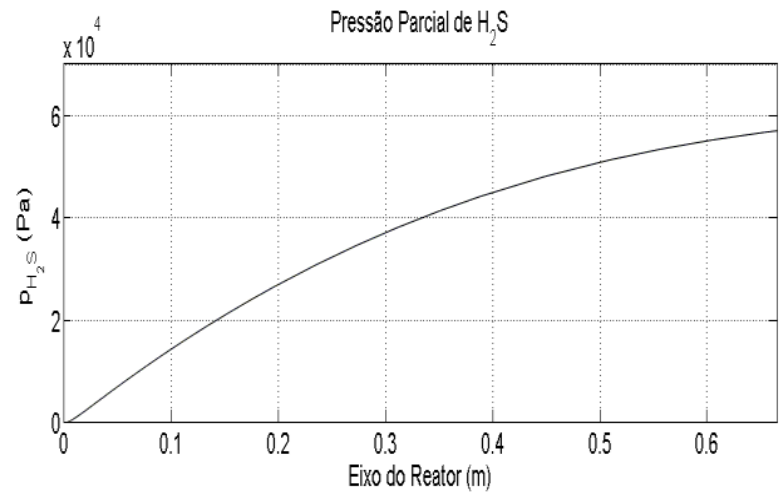

Figura 4 - Pressão parcial do sulfeto de hidrogênio.

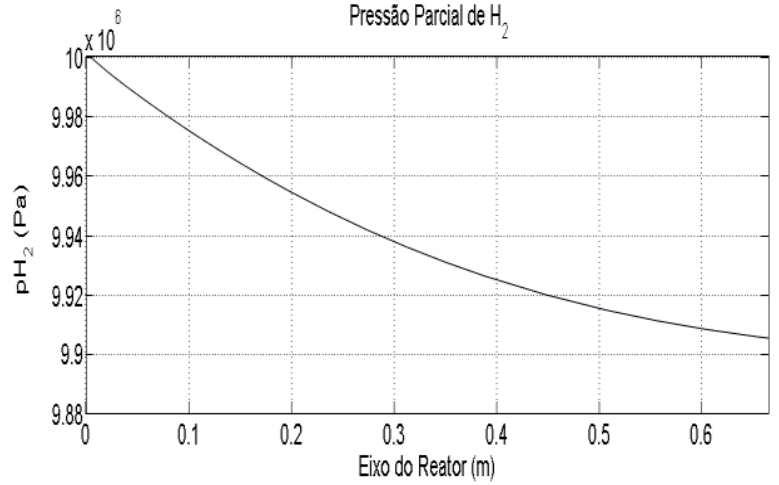

Figura 5 - Pressão parcial do hidrogênio.

\section{CONCLUSÕES}

A partir da análise dos resultados da simulação realizada, e comparando estes com os valores experimentais obtidos através de uma unidade de planta piloto, foi possível comprovar a validação do modelo empregado a esses dados. Os perfis de decaimento da concentração de enxofre ao longo do reator, bem como o comportamento da pressão parcial de hidrogênio, além das concentrações na superfície catalítica e na fase diluída para o sulfeto de hidrogênio e para o hidrogênio confirmam que o modelo é válido para previsão do comportamento da unidade de HDT.

É interessante destacar que, ao simular o processo para diferentes relações entre o óleo diesel e a carga de hidrogênio, à medida que esta relação aumentava, o sistema tendia a desviar-se do equilíbrio, e como consequência as concentrações na interface não eram corretamente calculadas. Isso se deve porque nos balanços, foi utilizada a lei de Henry, que 
usa a hipótese de equilíbrio entre as fases de agregação da matéria do sistema. Para corrigir este erro, deve-se utilizar o conceito de fugacidade nos balanços de massa para os componentes na fase gasosa.

\section{NOMENCLATURA}

\section{$a^{L}$ \\ $a^{s}$ \\ $\mathrm{Ci}$ \\ $C i^{S}$ \\ $D_{i}^{L}$ \\ $H_{i}$ \\ $k_{i}$ \\ $k_{i}^{s}$ \\ $R$ \\ $T$ \\ $\mathrm{T}_{\text {medio }}$ \\ $u^{G}$ \\ $u^{L}$ \\ $\boldsymbol{\vartheta}$ \\ Vn}

Letras Latinas

\section{Letras Gregas}

$\Delta \rho_{T}$

$\Delta \rho_{p}$

$\lambda i$

$\eta$

$\rho_{L}$

$\rho_{0}$

$\rho_{15,6}$

$\sigma_{H D S}$

$\xi$

$\mu$

$\epsilon$
Área específica da Transferência de massa gás-líquido

Área específica da Transferência de massa líquido-sólido

Concentração do componente i no líquido

Concentração do componente i no sólido

Difusividade Molecular

Coeficiente de Henry para o componente $i$

Coeficiente de Transferência de massa gás-

líquido do componente $i$

Coeficiente de Transferência de massa líquido-

sólido do componente $i$

Constante universal dos Gases

Temperatura

Temperatura média de bolha da mistura

Velocidade superficial do gás

Velocidade superficial do Líquido

Volume molar nas condições de operação

Volume molar nas CNTP

Influência da Temperatura na variação da densidade

Influência da pressão na variação da

densidade

Solubilidade do componente $i$

Efetividade Catalítica

Densidade do óleo diesel nas condições de operação

Densidade do óleo diesel nas CNTP

Densidade do óleo diesel a $15,6^{\circ} \mathrm{C}$

Coeficiente Estequiométrico do componente $i$

Fator de diluição

Viscosidade dinâmica

Porosidade do leito catalítico $\left[m^{2}\right]$

$\left[m^{2}\right]$

$\left[\mathrm{mol} / \mathrm{m}^{3}\right]$

$\left[\mathrm{mol} / \mathrm{m}^{3}\right]$

$\left[\mathrm{m}^{2} / \mathrm{s}\right]$

[]

$[\mathrm{m} / \mathrm{s}]$

$[\mathrm{m} / \mathrm{s}]$

$[\mathrm{J} / \mathrm{mol} \mathrm{K}]$

$[\mathrm{K}],\left[{ }^{\circ} \mathrm{C}\right],\left[{ }^{\circ} \mathrm{R}\right]$

$\left[{ }^{\circ} R\right]$

$[\mathrm{m} / \mathrm{s}]$

$[\mathrm{m} / \mathrm{s}]$

[ $\left.\mathrm{m}^{3} / \mathrm{mol}\right]$

$\left[\mathrm{m}^{3} / \mathrm{mol}\right]$

[]

[]

[Nl/kg.MPa]

[]

$\left[\mathrm{kg} / \mathrm{m}^{3}\right]$

$\left[\mathrm{kg} / \mathrm{m}^{3}\right]$

$\left[\mathrm{kg} / \mathrm{m}^{3}\right]$

[]

[]

[mPa S]

[] 


\section{REFERÊNCIAS}

AHMED, T. Hydrocarbon Phase Behauior. Gulf Publishing, Houston , 1989.

BONFÁ, M. H. P. Diesel S10: impacto sobre o rendimento do parque de refino brasileiro em 2020 e propostas mitigadoras, Rio de Janeiro: Dissertação de M.Sc., COPPE/UFRJ, 2011

CHOWDHURY, R.; PEDERNERA, E.; REIMERT, R. Trickle - bed reactor model for desulfurization and dearomatization of diesel. AIChE Journal, v.48, p.126-135, 2002.

FROMENT, G. F.; BISCHOFF, K. B. Chemical Reactor Analysis and Design. 2. ed. New York: Wiley, 1990.

GOTO, S.; SMITH, J. M. Trickle-bed Reactor Performance: I. Holdup and Mass Transfer Effects. AIChE Journal, v.21, p.706, 1975.

KORSTEN, H.; HOFFMANN, U. Three Phase Reactor Model for Hydrotreating in Pilot Trickle Bed Reactors. AiChe Journal, v.42, p.1350-1360, 1996.

NETO, A.T.P. Modelagem e Simulação de Reatores de Hidrotratamento de Diesel, Campina Grande, tese de D.Sc., UAEQ/UFCG, 2013.

PERRY, R. H.; GREEN, D. Peny's Chemical Engineers Handbook. 6 ${ }^{\text {a }}$ Ed. New York: McGrawHill, 1984.

REID, R. C.; PRAUSNITZ, J. M.; POLING, B. E. The Properties of Gases \& Liquids. $4^{\mathrm{a}}$ Ed. New York: McGraw-Hill, 1987.

SCHWEITZER, J. M.; LOPEZ-GARCIA, C.; FERRE, D. In-depth modeling of gas oil hydrotreating: From feedstock reconstruction to reactor stability analysis. Catalysis Today, v.150, p.279-299, 2010.

\section{AGRADECIMENTOS}

Os autores agradecem ao apoio financeiro provido pelo CNPq/UFCG e ao Laboratório de Experimentação Numérica de Processos (LENP) da Unidade Acadêmica de Engenharia Química (UAEQ/UFCG). 